\title{
Farthest-Point Queries with Geometric and Combinatorial Constraints
}

\author{
Ovidiu Daescu ${ }^{\mathrm{a}}$, Ningfang Mi ${ }^{\mathrm{a}}$, Chan-Su Shin ${ }^{\mathrm{b}}$, and Alexander Wolff ${ }^{\mathrm{c}}$ \\ a Department of Computer Science, University of Texas at Dallas, Richardson, TX 75083, USA \\ ${ }^{\mathrm{b}}$ School of Electronics and Information Engineering, Hankuk University of Foreign Studies, Korea \\ ${ }^{\mathrm{c}}$ Faculty of Computer Science, Karlsruhe University, Germany. WWW: i11www.ilkd.uka.de/algo/people/awolff
}

\section{Introduction}

In this paper we discuss farthest-point problems, in which a sequence $S=\left(p_{1}, p_{2}, \ldots, p_{n}\right)$ of $n$ points in the plane is given in advance and can be preprocessed to answer various queries efficiently. We first consider the general setting where query points can be arbitrary, then we investigate a special setting where each point in $S$ is queried exactly once.

To describe our problems, we use the following notation. Given two points $p \neq q$, let $p q$ denote the line through the points $p$ and $q$, let $\overline{p q}$ denote the line segment joining $p$ and $q$, and let $|p q|$ be the Euclidean distance of $p$ and $q$. We will use $\varepsilon$ to denote a fixed arbitrarily small positive number.

FARThestPoint AboveLine (FPAL): Given a pair $\left(q, l_{q}\right)$, where $q$ is a point and $l_{q}$ is a line through $q$, decide whether there is a point in $S$ above $l_{q}$, and if yes report the one farthest from $q$.

Our solution to this problem is a data structure based on [6] that takes $O(n \log n)$ space, $O\left(n^{1+\varepsilon}\right)$ preprocessing time and $O\left(n^{1 / 2+\varepsilon}\right)$ query time. We can do better if $S$ is in convex position:

FPALinConvexPoLyGON (FPALCP): Given a convex $n$-gon $C$ and a pair $\left(q, l_{q}\right)$, where $q$ is a point and $l_{q}$ is a line through $q$, decide whether there is a point in $C$ above $l_{q}$, and if yes report the one farthest from $q$.

Our solution takes $O(n \log n)$ space, $O\left(n \log ^{2} n\right)$ preprocessing time and $O\left(\log ^{2} n\right)$ query time.

FARThestPointSameSide (FPSS): Given a triplet $\left(q, \mathcal{L}_{q}, \Delta\right)$, where $q$ is a point and $\mathcal{L}_{q}$ is a line through $q$ such that all points in $S$ are within distance $\Delta$ from $\mathcal{L}_{q}$, decide whether there is a

Email addresses: daescu@utdallas.edu (Ovidiu Daescu), nxm024100@utdallas.edu (Ningfang Mi), cssin@hufs.ac.kr (Chan-Su Shin). point $p \in S$ such that (i) $|q p|>\Delta$, and (ii) $p$ is above the line $l_{q}$ orthogonal to $\mathcal{L}_{q}$ at $q$. If yes, report the point $p$ farthest from $q$ that fulfills (ii).

Our data structure for this problem has the same time and space bounds as that for FPALCP.

FARTHESTINDEXEDPOINTOTHERSIDE (FIPOS): Given a triplet $(i, j, \Delta)$, such that $1 \leq i<j \leq n$ and all points $p_{k} \in S$ with $i<k<j$ are within distance $\Delta$ from $p_{i} p_{j}$, decide whether there is a point $p_{k}$ with $i<k<j$ such that (i) $\left|p_{i} p_{k}\right|>\Delta$, and (ii) $p_{k}$ and $p_{j}$ lie on different sides of the line that goes through $p_{i}$ and is orthogonal to $p_{i} p_{j}$. If yes, report the point $p_{k}$ farthest from $p_{i}$ that fulfills (ii).

Our time and space bounds for this problem are by a log-factor above those for FPALCP.

In the special setting where each point in $S$ is queried exactly once we investigate the following problem. We assume the existence of a point $p \notin S$.

BatchedFarthestIndexedPointSameSide (BFIPSS): For each point $p_{i} \in S$ decide whether there is a point $p_{f} \in\left\{p_{1}, \ldots, p_{i}\right\}$ that lies on the same side as $p$ with respect to the perpendicular bisector of $p$ and $p_{i}$. If yes report the point $p_{f}$ farthest from $p$ that has the above property.

Our algorithm for this problem runs in $O\left(n \log ^{2} n\right)$ time and uses $O(n \log n)$ space.

The problems we study are related to the nearest-point query problem, to the all-pairs farthest and closest neighbors problem, and to the closest-point-to-line query problem. Although these problems are well understood, we are not aware of work on the problems we consider.

Applications of our data structures are polygonal chain approximation [2], approximate solutions for the one-cylinder problem [1], and geometric spanning trees [5]. 


\section{0th European Workshop on Computational Geometry}

\section{Farthest-Point Queries}

We first tackle FPALCP, since it is part of the solutions for the problems FPSS and FIPOS.

In the preprocessing phase, we construct a balanced binary tree $T$ in $O\left(n \log ^{2} n\right)$ time as follows. The vertices of the convex polygon $C$, in counterclockwise order from the rightmost vertex, are associated with the leaves of $T$. At each internal node $u$, we compute and store the convex hull and the farthest-point Voronoi diagram $V_{u}$ of the leaf descendants of $u$ from the information available at the children of $u$. We then preprocess $V_{u}$ for planar point-location queries, which takes a total of $O(n \log n)$ time for each level of $T$. Thus, the overall computation of $T$ takes $O\left(n \log ^{2} n\right)$ time.

Given a query pair $\left(q, l_{q}\right)$, we find in $O(\log n)$ time the intersection points of $l_{q}$ with the boundary $\partial C$ of $C$ by binary search. We assume that $l_{q}$ has non-empty intersection with the interior of $C$ (the four other cases are trivial). The sought point is either one of the two intersection points of $l_{q}$ with $\partial C$ or a vertex of $C$ that is above $l_{q}$. Without loss of generality, we assume that no vertex of $C$ lies on $l_{q}$ and that $l_{q}$ has positive slope.

We query $T$ as follows. We select the two endpoints of the segments intersected by $l_{q}$ that are above $l_{q}$. Let $s$ be the first and $t$ the second endpoint in counter-clockwise order on $\partial C$. We walk in $T$ from $s$ to $t$ and collect a set $\mathcal{V}$ of $O(\log n)$ farthest-point Voronoi diagrams in two phases. In the ascending phase we go upwards from $s$. Whenever we get to a node $u$ from its left child, we add to $\mathcal{V}$ the Voronoi diagram stored at the right child of $u$. In the descending phase we go down towards $t$. Whenever we go to the right child of a node $u$, we add to $\mathcal{V}$ the Voronoi diagram stored at the left child of $u$. Clearly, all points associated with these Voronoi diagrams are above $l_{q}$ and thus the sought vertex is either $s, t$ or one of these points. We locate $q$ in $O(\log n)$ time in each Voronoi diagram in $\mathcal{V}$ and keep track of the point farthest from $q$. Thus we can answer the query in $O\left(\log ^{2} n\right)$ time.

Theorem 1 There is a data structure for FPALCP that takes $O(n \log n)$ space, $O\left(n \log ^{2} n\right)$ preprocessing time and $O\left(\log ^{2} n\right)$ query time.

One can answer queries for FPSS using the same approach as for FPAL: construct a partition tree based on a fine simplicial partition in $O\left(n^{1+\varepsilon}\right)$ time [6] and enhance it with a second-level data

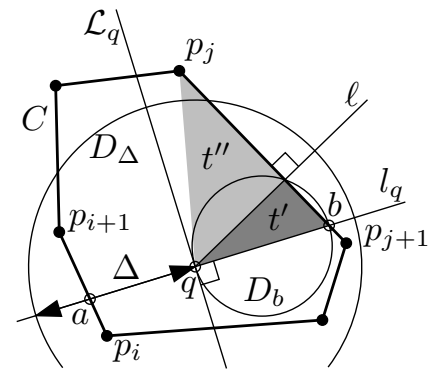

Fig. 1. The point $p$ farthest from $q$ must be a vertex of the convex hull $C$ of $S$ if $|q p|>\Delta$.

structure consisting of the farthest-point Voronoi diagram (of the points at each internal node) preprocessed for planar point location. This data structure can be used to obtain a collection of disjoint subsets of points representing all points in $S$ which are above the line $l_{q}$, and then query the farthest-point Voronoi diagrams for the points in each subset in order to answer the query. Note that the point farthest from the query point $q$ may lie inside the convex hull $C$ of $S$, so it seems our solution of FPALCP cannot be applied here. The following lemma, however, does give us a way to use the FPALCP data structures to solve FPSS.

Lemma 2 Given $S \subset \mathbb{R}^{2}$ and a triplet $\left(q, \mathcal{L}_{q}, \Delta\right)$, where $q$ is a point and $\mathcal{L}_{q}$ is a line through $q$ such that all points in $S$ are within distance $\Delta$ from $\mathcal{L}_{q}$, if there is a point $p \in S$ such that (i) $|q p|>\Delta$, and (ii) $p$ is above the line $l_{q}$ orthogonal to $\mathcal{L}_{q}$ at $q$, then the point in $S$ farthest from $q$ is a vertex of the convex hull $C$ of $S$.

PROOF. Wlog. we assume that $l_{q}$ intersects $\partial C$ in two line segments $\overline{p_{i} p_{i+1}}$ and $\overline{p_{j} p_{j+1}}$ with $1 \leq$ $i<j<n$ and that $p_{i+1}, \ldots, p_{j}$ all lie above $l_{q}$. Let $a$ and $b$ be the intersection points. Clearly for each triangle $q p_{k} p_{k+1}$ above $l_{q}$ either $p_{k}$ or $p_{k+1}$ is farthest from $q$. We now consider the triangle $t=$ $q p_{j} b$, the triangle $q a p_{i+1}$ is analogous.

Let $\ell$ be the line through $q$ that is orthogonal to $p_{j} p_{j+1}$. Consider the right-angled triangle $t^{\prime}$ (shaded dark in Figure 1) that is defined by $\ell$, $p_{j} p_{j+1}$ and $q b$. Due to Thales' theorem $t^{\prime}$ is contained in the disk $D_{b}$ whose diameter is $\overline{q b}$. Since $|q b| \leq \Delta, D_{b}$ (and thus $t^{\prime}$ ) is contained in the radius- $\Delta$ disk $D_{\Delta}$ centered at $q$.

Now if $q_{j} \in t^{\prime}$ then $t \subseteq t^{\prime} \subseteq D_{\Delta}$. Otherwise $\ell$ splits $t$ into $t^{\prime}$ and another right-angled triangle $t^{\prime \prime}$ (shaded lightly in Figure 1) that is defined by $q p_{j}$, $p_{j} p_{j+1}$ and $\ell$. Since $\overline{q p_{j}}$ is the hypothenuse of $t^{\prime \prime}$, 
$p_{j}$ is farthest from $q$ in $t^{\prime \prime}$. Thus the point farthest from $q$ either lies in $D_{\Delta}$ or is a vertex of $C$.

Theorem 3 There is a data structure for FPSS that takes $O(n \log n)$ space, $O\left(n \log ^{2} n\right)$ preprocessing time and $O\left(\log ^{2} n\right)$ query time.

To solve FIPOS, the indexed version of FPSS, in the preprocessing phase we construct an $O\left(n \log ^{2} n\right)$-size balanced binary tree $T$ as follows. Each leaf of $T$ is associated with a point in $S$ such that the point $p_{i} \in S$ is stored at the $i$-th leaf of $T$. We go up the tree $T$ and, at each internal node $v$, we compute and store the convex hull $C_{v}$ of the leaf descendants of $v$. We also compute and store at $v$ a secondary level data structure in the form of a balanced binary tree $T_{v}$ of the farthest-point Voronoi diagrams on the vertices of $C_{v}$, enhanced with a planar point-location data structure, similar to the one used in solving FPSS. Then, the overall computation of $T$ takes $O\left(n \log ^{3} n\right)$ time and requires $O\left(n \log ^{2} n\right)$ space. For a query pair $(i, j)$, let $\pi_{i j}$ be the path in $T$ from $p_{i}$ to $p_{j}$. We use this path to obtain a set $\mathcal{C}$ of $O(\log n)$ convex hulls whose union contains only the points $p_{k}$ with $i<k<j$ as follows. In the ascending phase we add to $\mathcal{C}$ the convex hull stored at the right child of $v$ if $\pi_{i j}$ gets to $v$ from its left child. In the descending phase we add to $\mathcal{C}$ the convex hull stored at the left child of $v$ if $\pi_{i j}$ goes from $v$ to its right child. For each convex hull $C \in \mathcal{C}$, querying the secondary data structure reduces to FPSS. Let $t$ be the number of vertices on $C$. Then we can determine in $O\left(\log ^{2} t\right)$ time whether there is a $k$, $i<k<j$, such that the point $p_{k} \in S$ associated with $C$ satisfies the two FIPOS conditions. Since the size of the set $\mathcal{C}$ is $O(\log n)$, the overall query time is $O\left(\log ^{3} n\right)$.

Theorem 4 There is a data structure for FIPOS that takes $O\left(n \log ^{2} n\right)$ space, $O\left(n \log ^{3} n\right)$ preprocessing time and $O\left(\log ^{3} n\right)$ query time.

Building on our solutions of FPALCP, FPSS, and FIPOS, we extend a recent result in [3].

Theorem 5 Given a polygonal chain $P=\left(p_{1}, p_{2}\right.$, $\left.\ldots, p_{n}\right)$ in the plane, the min-\# problem with the tolerance zone criterion and $L_{2}$ distance metric can be solved in $O\left(F(m) n \log ^{3} n\right)$ time with $O\left(n \log ^{2} n\right)$ space, where $F(m)$ is the number of vertices of the path approximation graph reachable from $p_{1}$ with at most $m-2$ edges and $m$ is the number of vertices of an optimal approximating path.
A version of BFIPSS without the index restriction has been considered in [5]. There the problem was to report for each point $p_{i} \in S$ a point farthest from the fixed point $p \notin S$ that lies on the same side as $p$ with respect to the perpendicular bisector of $p$ and $p_{i}$. In [5] the problem is reduced to the problem of finding for each $p_{i} \in S$ the first disk in a sequence of disks that does not contain $p_{i}$. This problem has been addressed in [2] under the name off-line ball exclusion search (OLBES). The authors set up a tree data structure with a space requirement of $O(n \log n)$ and then query this structure with each point in $S$. This results in a total running time of $O(n \log n)$ for OLBES in dimension $d=2$. For $d>2$ the problem is solved differently in $O\left(n^{2-2 /(\lfloor d / 2\rfloor)+1}\right)$ time. In [5], a version of OLBES where all disks intersect a common point has been solved for $d=2$ in $O(n \log n)$ time and $O(n)$ space by sweeping an arrangement of circular arcs. To solve BFIPSS in dimension 2 we set up a tree data structure similar to [4, proof of Lemma 2]. Here, however, we must solve a different OLBES problem in each query and thus need to modify our tree successively.

Theorem 6 BFIPSS can be solved in $O\left(n \log ^{2} n\right)$ time and $O(n \log n)$ space for a sequence $S$ of $n$ points and a point $p \notin S$ in the plane.

PROOF. Let $D_{n+1}=\emptyset$ and let $D_{1}, \ldots, D_{n}$ be the sequence of disks in order of non-increasing radius that are centered on the points in $S$ and touch $p$. We build a binary tree $\mathcal{B}$ that we query with the points in $S$, and the answer of a query will correspond to the index of the first disk in the sequence $D_{1}, \ldots, D_{n+1}$ that does not contain the query point. The leaves of $\mathcal{B}$ correspond to these answers from left to right. Each inner node $v$ stores the intersection $I_{v}$ of all disks (except $D_{n+1}$ ) that correspond to the leaves in the subtree rooted at $v$. We label each node $v$ with a pair $\left[a_{v}, b_{v}\right]$ encoding the set $S_{v}=\left\{a_{v}, \ldots, b_{v}\right\}$ of consecutive indices that correspond to these disks. In Figure 2 a tree with $n=13$ is depicted. We build $\mathcal{B}$ in a bottomup fashion. Each inner node has two children in the previous level, except possibly a level's rightmost node that can have a right child in an earlier level, see the node with label $[9,13]$ in Figure 2.

Querying $\mathcal{B}$ with a query point $q$ means to follow a path from the root to a leaf. In each inner node $v$ with left child $\ell$ the test $q \in I_{\ell}$ is performed. If $q \in$ 


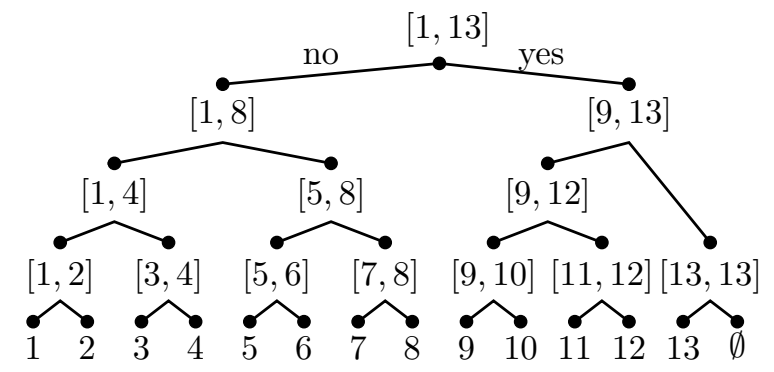

Fig. 2. The tree data structure $\mathcal{B}$ for $n=13$.

$I_{\ell}$, the query continues with the right, otherwise with the left child of $v$.

Other than in $[2,4]$, we start with an empty skeleton of $\mathcal{B}$, i.e. all inner nodes $v$ are labeled by $\left[a_{v}, b_{v}\right]$, but all intersections $I_{v}$ are set to $\mathbb{R}^{2}$. Also other than in [4], the order in which we query becomes crucial. We go through the points $p_{1}, \ldots, p_{n} \in S$ in order of increasing index. Before querying $\mathcal{B}$ with $p_{i}$ we update $\mathcal{B}$ by adding the new disk $D_{k}$ centered on $p_{i}$ (note that usually $k \neq i$ ) to the intersection $I_{v}$ for each node $v$ on the path from the root to the leaf that corresponds to $D_{k}$.

The result of a query with $p_{i}$ is the disk $D_{j}$ that corresponds to the leaf at the end of the query path $\pi$. If $j=n+1$ then $\pi$ is the rightmost root-leaf path. Consider the left children of the nodes on $\pi$. The sets $S_{\ell}$ that belong to these left children partition $\{1, \ldots, n\}$. In other words, the intersection of $I_{\ell}$ over these children is $D_{1} \cap \cdots \cap D_{n}$. Since $\pi$ is the rightmost root-leaf path, the containment queries in all nodes on $\pi$ were answered positively. Thus $q_{i}$ is contained in all disks currently in $\mathcal{B}$, i.e. $q_{i} \in D\left(q_{1}, p\right) \cap \cdots \cap D\left(q_{i}, p\right)$, where $D(a, b)$ is the disk centered at $a$ that touches $b$. This means that none of $q_{1}, \ldots, q_{i}$ lies in the halfplane $h\left(p, q_{i}\right)$ that contains $p$ and whose boundary is the perpendicular bisector of $p$ and $q_{i}$. Otherwise [5, Lemma 1] would guarantee that $q_{i} \notin D\left(q_{k}, p\right)$ for the point $q_{k}$ in $\left\{q_{1}, \ldots, q_{i}\right\}$ farthest from $p$ in $h\left(p, q_{i}\right)$.

If $j \leq n$ we again consider the left children of the nodes on the query path $\pi$ of $q_{i}$. The sets $S_{\ell}$ partition $\{1, \ldots, j-1\}$ if we take only those left children $\ell$ into account that do not themselves lie on $\pi$. Similarly to above, the intersection of $I_{\ell}$ over these children is $D_{1} \cap \cdots \cap D_{j-1}$. Thus $q_{i}$ is contained in all $D_{k}$ with $k<j$ that are currently in $\mathcal{B}$. On the other hand, since $\pi$ is not the rightmost root-leaf path, there must be left children that lie on $\pi$. The last such left child $v$ is the root of the subtree whose rightmost leaf corresponds to $D_{j}$. Thus $v$ is asso- ciated with some set $S_{v}=\left\{i_{v}, \ldots, j\right\}$, where $1 \leq$ $i_{v} \leq j$. Since we have already observed that $q_{i}$ is contained in all $D_{k}$ with $k<j$ that are currently in $\mathcal{B}$, but $\pi$ came to $v$ via a "no"-branch, we now know that $q_{i} \notin D_{j}$. Let $m$ be such that $D_{j}=D\left(q_{m}, p\right)$. Note that $q_{i} \notin D\left(q_{m}, p\right)$ means that $D\left(q_{m}, p\right)$ was inserted in $\mathcal{B}$ before querying with $q_{i}$, and thus $m \leq i$. Since $q_{i} \notin D\left(q_{m}, p\right)$, and $q_{i} \in D\left(q_{r}, p\right)$ for all $r \leq i$ with $\left|p q_{r}\right|>\left|p q_{m}\right|$, [5, Lemma 1] yields that $q_{m}$ is farthest from $p$ in $\left\{q_{1}, \ldots, q_{i}\right\} \cap h\left(p, q_{i}\right)$.

The running time is as follows. Querying $\mathcal{B}$ takes $O\left(\log ^{2} n\right)$ time since the height of $\mathcal{B}$ is $O(\log n)$ and in each node of the query path the query point has to be located in the intersection $I_{v}$ of some disks, which can be done in $O(\log n)$ time.

When we update $\mathcal{B}$ by adding a new disk $D_{j}$, we have to go from the root to the leaf that corresponds to $D_{j}$. In each node on this path we must compute $I_{v} \cap D_{j}$ and update our data structure for $I_{v}$. This can be done in $O(\log n)$ time per node by a procedure detailed in [5, proof of Lemma 4]. Thus each update also takes $O\left(\log ^{2} n\right)$ time.

Now the running time of $O\left(n \log ^{2} n\right)$ is obvious. The space consumption is $O(n \log n)$ since a) each disk contributes only to intersections stored on the path from the root to "its" leaf, and b) a disk that contributes to some intersection $I_{v}$ adds at most one arc to the boundary of $I_{v}[5$, Fact 2].

\section{References}

[1] M. Bădoiu, S. Har-Peled, and P. Indyk. Approximate clustering via core-sets. In Proc. STOC'02, pages 250$257,2002$.

[2] D. Z. Chen, O. Daescu, J. Hershberger, P. M. Kogge, and J. Snoeyink. Polygonal path approximation with angle constraints. In Proc. SODA'01, pages 342-343, Washington, D.C., 2001.

[3] O. Daescu and N. Mi. Polygonal path approximation: A query based approach. In Proc. ISAAC'03, volume 2906 of $L N C S$, pages 36-46, 2003. Springer.

[4] J. Gudmundsson, H. Haverkort, S.-M. Park, C.-S. Shin, and A. Wolff. Facility location and the geometric minimum-diameter spanning tree. In Proc. APPROX '02, vol. 2462 of $L N C S$, pages 146-160, 2002. Springer.

[5] J. Gudmundsson, H. Haverkort, S.-M. Park, C.-S. Shin, and A. Wolff. Facility location and the geometric minimum-diameter spanning tree. Computational Geometry: Theory and Appl., 27(1):87-106, 2004.

[6] J. Matoušek. Efficient partition trees. Discrete and Computational Geometry, 8:315-334, 1992. 